Andreas Schapowal

E rkältungskrankheiten, Infektionen der oberen und unteren Atemwege stellen in der pädiatrischen Praxis bewährte Indikationen für Phytotherapeutika dar. Grundsätzlich gelten für Phytotherapeutika dieselben Bewertungskriterien wie für synthetische Arzneimittel hinsichtlich Wirkungen, Nebenwirkungen und Dosisfindungen. Viele pflanzliche Heilmittel sind allerdings über Jahrhunderte angewandt worden und haben sich bewährt, ohne dass nennenswerte Nebenwirkungen auftraten. Das gilt z.B. für Kamillen-, Linden- und Holunderblüten. Derartige Phytotherapeutika sind zur Selbstmedikation durchaus geeignet. Die Kommission E des ehemaligen Bundesgesundheitsamtes hat als Expertengruppe für Phytopharmaka in Deutschland von 1978-1995 das wissenschaftliche Material über zahlreiche pflanzliche Heilmittel in Monographien gesammelt und bewertet; sie hat jedoch keine Hinweise auf die Dosierungen bei Kindern gegeben.

Dosierungsangaben haben in der Kinderheilkunde grundsätzlich als Bezugswerte Körpergewicht, -oberfläche und/oder Lebensalter zu berücksichtigen. Bei Säuglingen und Kleinkindern richtet sich die Dosierung in der Regel nach dem Körpergewicht, bei älteren Kindern nach der Körperoberfläche oder dem Alter. Nach der «Note for Guidance on Clinical Investigation of Medicinal Products in Children» können Kinderdosierungen auf der Basis von Körpergewicht und Körperoberfläche aus der Erwachsenendosis extrapoliert werden [1]. Als Faustregel gilt, dass Kinder von 6-9 Jahren etwa die Hälfte, Kinder von 10-12 Jahren zwei Drittel der Erwachsenendosis erhalten.

\title{
Phytopharmaka bei Atemwegsinfekten in der Pädiatrie
}

Die Wirksamkeit von zahlreichen Phytotherapeutika ist aus der Erfahrungsmedizin bekannt und schlägt sich in den Monographien nieder. Hingegen sind klinische Studien mit standardisierten Extrakten eher rar, so dass die Dosisfindung bei Kindern meist auf der Basis von Körpergewicht und -oberfläche aus der Erwachsenendosis extrapoliert wird. Der Übersichtsartikel zeigt die Wirkungen und Indikationen von Phytotherapeutika bei Atemwegsinfekten auf und gibt Dosierungshinweise anhand von Monographien und Studien.

\section{Wirkungen und Darreichungsformen}

Phytotherapeutika wirken bei Atemwegserkrankungen in erster Linie symptomatisch (Tab. 1); einige Substanzen haben spezifische kausale Wirkungen (Tab. 2). Bei den ersten Infektzeichen kommen als Darreichungsform heisse Erkältungstees sowie Bäderextrakte für ansteigende Fuss- und Armbäder infrage. Bei Entzündungen in der Mundhöhle und im Pharynx können Pastillen, Lutschtabletten und Gurgellösungen gegeben werden. Für Infekte der oberen Atemwege eignet sich besonders die Inhalation von ätherischen Ölen, für die unteren Atemwege Hustentropfen oder -sirup als ethanolischwässrige Tinkturen. Trockenextrakte als Tabletten, Dragées oder Kapseln stehen für Sinusitis und Bronchitis zur Verfügung.

\section{Arzneipflanzen und ihre Indikationen bei Atemwegserkrankungen}

\section{Aromatika}

Ätherische Öle enthalten Gemische aus phenolischen Verbindungen, Terpenen und Phenylpropanverbindungen. Aus folgenden Pflanzen werden aromatische Arzneidrogen hergestellt: Anis, Campher, Eukalyptus, Fenchelfrüchte, Fichtennadel, Grindeliakraut, Kamillenblüten, Kiefernnadeln, Lärchenter- pentin (gewonnen durch Anbohren der Stämme), Pfefferminze, Quendelkraut, Salbeiblätter, Sternanisfrüchte, Thymiankraut.

Ätherische Öle können antiphlogistisch, antibakteriell, antimykotisch, bronchospasmolytisch, expektorierend und sedativ wirken. Sie beeinflussen Enzyme, Ionenkanäle und Rezeptoren der Zellmembranen und wirken membranstabilisierend. Indikationen sind banale Infekte, Husten, Bronchitis, Sinusitis (Kamillenblüten), virale Tonsillitis (Salbei).

\section{Saponindrogen}

Saponindrogen senken die Oberflächenspannung des Sputums, stimulieren die Flimmerzellen, wirken sekretolytisch, sekretomotorisch, antiphlogistisch, bakteriostatisch, antimykotisch. Sie werden v.a. bei Husten und Bronchitis eingesetzt. Folgende pflanzliche Drogen werden verwendet: Efeublätter, Primelwurzel, Sanikelkraut, Schlüsselblumenblüten, Rote Seifenwurzel, Senegawurzel, Süssholzwurzel.

\section{Schleimstoffdrogen}

Schleimstoffdrogen enthalten Polysaccharide aus Monosacchariden, Glukuron-, Galakturonsäuren und Arabinogalaktanen. Sie sind hydrophil und bilden bei Kontakt mit Wasser durch Aufquellen abdeckende, kolloidale Lösungen. Sie wirken schleimhautprotektiv, reizlindernd und antiphlogistisch. Indikationen sind Husten, Bronchitis, Tonsillitis. Verwendete pflanzliche Drogen 
sind Eibischblätter und -wurzel, Huflattichblätter, Isländisches Moos, Malvenblätter und -blüten, Spitzwegerichkraut und Wollblumen.

\section{Schweisstreibende Drogen}

Holunderblüten (Sambuci flos) und Lindenblüten (Tiliae flos) sind die wichtigsten schweisstreibenden Drogen. Sie werden als heisse Tees beim banalen Infekt eingesetzt. Zur Schwitzkur gehören ein heisses Bad mit langsam steigender Temperatur und anschliessendes Liegen, eingehüllt in warme Decken.

\section{Fiebersenkende Drogen}

Mädesüssblüten und -kraut (Spireae flos/herba) und Weidenrinde (Salicis cortex) wirken antipyretisch.

Weisser Senfsamen (Sinapis albae semen) fördert die Durchblutung und wird in Form des Senfmehlfussbades bei Beginn der Erkältung eingesetzt.

\section{Senfmehlfussbad}

Fussbäder mit ätherischen Ölen wirken reflektorisch auf den gesamten Organismus und fördern die Durchblutung. Sie sind auch für Kinder geeignet und sollten sofort zu Beginn einer Erkältung eingesetzt werden: $3 \mathrm{EL}$ Senfmehl mit 2 I warmem Wasser $\left(37^{\circ} \mathrm{C}\right)$ vermengen und ca. 15 Min. die Füsse darin baden. Anschliessend lauwarm abspülen, warme Wollsocken anziehen und nachruhen.

\section{Spezielle Dosierungs- empfehlungen bei Kindern}

Im Folgenden werden Dosierungsrichtlinien für die Pädiatrie von einigen ausgewählten Arzneipflanzen in alphabetischer Reihenfolge gegeben. Quellen sind die jeweiligen zur Verfügung stehenden Monographien sowie drei gute und als Nachschlagewerke geeignete Lehrbücher $[2,3,4,5]$. Auf die Empfehlung von Handelspräparaten wird bewusst verzichtet.

\section{Althaeae folium (Eibischblätter) und Altheae radix (Eibischwurzel)}

Indikationen sind Schleimhautreizungen im Mund- und Rachenraum und damit verbundener trockener Reizhusten [6]. Nebenwirkungen und Risiken

Tab. 1. Symptomatische Wirkungen von Phytopharmaka bei Atemwegserkrankungen

\begin{tabular}{|ll|}
\hline schleimhautabdeckend, indirekt antitussiv & $\begin{array}{l}\text { Schleimdrogen wie z.B. Eibischwurzel, } \\
\text { Malve, Isländisch Moos }\end{array}$ \\
\hline sekretolytisch, sekretomotorisch & $\begin{array}{l}\text { Saponindrogen wie z.B. Efeu, Primel, } \\
\text { Schlüsselblume }\end{array}$ \\
\hline antiphlogistisch & z.B. Cineol, Kamillenblüten, Myrtol \\
\hline bronchospasmolytisch & z.B. Campher \\
\hline
\end{tabular}

Tab. 2. Kausale Wirkungen von Phytopharmaka bei Atemwegserkrankungen

\begin{tabular}{|ll|}
\hline virustatisch & z.B. Thymiankraut \\
\hline antibakteriell & z.B. Anis-, Kamillen-, Minz-, Thymianöl \\
\hline immunstimulierend & Eibischwurzel, Kamillenöl, \\
& Pelargonium-sidoides-Wurzel, \\
& Roter Sonnenhut, Sonnentaukraut \\
\hline
\end{tabular}

sind nicht bekannt. Anwendungsart in der Pädiatrie: Teezubereitungen. Die Empfehlungen für die Tagesdosierungen betragen für Eibischblätter 1-2 g bei Kindern >1-4 Jahre, 2-3 g >4-10 Jahre und 3-5 g >10-16 Jahre. Für die Eibischwurzel gelten folgende Dosierungsempfehlungen: $1,5-3 \mathrm{~g}$ bei $>1-4$ Jahre, 3-4 g >4-10 Jahre und 4-6 g >10-16 Jahre.

\section{Anisi fructus (Anis)}

Indikation: Katarrhe der Atemwege bei äusserer Anwendung in Form des ätherischen Öls [7]. Die Dosierung beträgt 1-2\%iges ätherisches Öl von 0-1 Jahre und 5-10\%ig von >1-16 Jahren.

\section{Anisi stellati fructus (Sternanisfrüchte)}

Die zerkleinerte Droge aus Sternanis ist indiziert für Katarrhe der Atemwege [8] und eignet sich für Aufgüsse sowie andere galenische Zubereitungen zur peroralen Applikation. Risiken, Gegenanzeigen und Wechselwirkungen sind nicht bekannt. Kinder von 0-1 Jahr nehmen 0,2-0,6 g Droge, >1-4 Jahre 0,6-1,2 g, >4-10 Jahre 1-2 g, >10-16 Jahre 1,5-3 g.

\section{Arnicae flos (Arnikablüten)}

Arnikablüten eignen sich zur äusseren Anwendung bei Prellungen und Stauchungen, im HNO-Bereich zum Mundspülen und Gurgeln bei Entzündungen der Schleimhäute und des Rachens [9]. Gegenanzeige ist eine Arnika-Allergie; als Allergene kommen Helenalin und seine Ester infrage. Zur Mundspülung wird eine $10 \%$ ige Tinktur verdünnt mit Wasser für Kinder >10-16 Jahre empfohlen.

Calendulae flos (Ringelblumenblüten) Die zerkleinerte Droge aus Ringelblumenblüten ist indiziert zur Bereitung von Aufgüssen sowie anderen galenischen Zubereitungen bei Entzündungen der Mund- und Rachenschleimhaut [10]. Die Dosierung beträgt für Kinder von 0-1 Jahr 1-2 g, >1- 4 Jahre 2-4 g, >4-16 Jahre 2-5 g.

\section{Caryophylli flos (Gewürznelken)}

Entzündliche Veränderungen der Mundund Rachenschleimhäute können auch mit Gewürznelken und daraus hergestelltem ätherischen Öl als Mundwasser behandelt werden [11]. Die Dosierung beträgt für >1-4 Jahre 1-2\%iges ätherisches Öl, >4-16 Jahre 1-5\%.

\section{Echinaceae purpureae herba (Purpursonnenhutkraut)}

Indikation ist die kurzfristige Behandlung von Infekten der Atemwege und der ableitenden Harnwege bei oraler Anwendung sowie schlecht heilende, oberflächliche Wunden bei topischer Anwendung [12]. Inhaltsstoffe sind Polysaccharide, Echinacosid, Cichoriensäure. Die orale Einnahme wurde von der Kommission E auf 2-3 Wochen beschränkt. Die Dosierung beträgt für Kinder >1-4 Jahre $3 \mathrm{ml}$ Presssaft, $>4-10$ Jahre $3-5 \mathrm{ml}$ und $>10-16$ Jahre 6-9 ml. Bei topischer Anwendung ist 
die Dosierungsempfehlung von >1-16 Jahren 15\%iger Presssaft. Die orale Anwendung wird in aller Regel sehr gut vertragen. Bei parenteraler Anwendung sind dosisabhängig Schüttelfrost, kurzfristige Fieberreaktionen, Übelkeit und Erbrechen beschrieben, ferner in Einzelfällen Reaktionen vom Soforttyp, im Besonderen bei bekannten Allergien auf Korbblütler.

\section{Eucalypti folium (Eukalyptusblätter)} Indikation für Eukalyptusblätter sind Erkältungskrankheiten der Luftwege [13]. In der Pädiatrie eignet sich besonders ätherisches Öl als Tropfen auf das Kopfkissen (>2-16 Jahre 4-6 Tr.). Wegen der möglichen Auslösung des Kratschmer-Reflexes (Atemstillstand) sind Anwendungen von ätherischen Ölen in Kopfnähe bei Kindern unter 2 Jahren kontraindiziert.

\section{Farfarae folium (Huflattichblätter)}

Indikationen laut Monographie sind akute Katarrhe der Luftwege mit Husten und Heiserkeit sowie akute entzündliche Erkrankungen der Mundund Rachenschleimhaut [14]. Die Tagesdosis beträgt 4,5-6 g Droge. In Huflattichblättern sind lebertoxische Pyrrolizidinalkaloide enthalten. Bei Huflattichtee darf die Tagesdosis nicht mehr als $10 \mu \mathrm{g}$ dieser Alkaloide enthalten, bei Extrakten und Frischpflanzenpresssaft nicht mehr als $1 \mu \mathrm{g}$. Die Anwendungsdauer ist auf 4-6 Wochen/ Jahr zu beschränken. Besser ist es, die Huflattich-Zuchtsorte anzuwenden, die frei von Pyrrolizidinalkaloiden ist.

\section{Filipendulae ulmariae flos (Mädesüss)}

Die zerkleinerte Droge und andere galenische Zubereitungen aus Mädesüss sind für Teeaufgüsse zur unterstützenden Behandlung bei Erkältungskrankheiten geeignet [15]. Bei Salicylat-Intoleranz sollte Mädesüss nicht angewandt werden. Die Dosierung beträgt bei Kindern $>1-4$ Jahre 1-2 g, >4-10 Jahre 2-3 g und >10-16 Jahre 2,5-3,5g.

\section{Foeniculi fructus (Fenchelfrüchte)}

Bei Katarrhen der oberen Luftwege können Fenchelfrüchte zerstossen, als Fenchelsirup oder als -honig gegeben werden [16]. Die Dosierung für Foeniculi fructus contusus beträgt für 0-1 Jahr 1-2 g, für >1-4 Jahre 1,5-3 g, für $>4-10$ Jahre 3-5 g und für >10-16 Jahre 5-7 g. Für Fenchelsirup oder honig sind die Dosierungen von $>1-4$ Jahre 3-6 g, >4-10 Jahre 6-10 g und >10-16 Jahre 10-20 g.

Hederae helicis folium (Efeublätter) Indikationen laut Monographie sind Katarrhe der Luftwege und symptomatische Behandlung chronisch-entzündlicher Bronchialerkrankungen [17]. Die Wirksamkeit eines EfeublätterTrockenextrakts bei chronischer Bronchitis wurde versus Ambroxol nachgewiesen [18]. Die Wirksamkeit bei Asthma wurde in einer randomisierten, doppelblinden, placebokontrollierten Cross-over-Studie von MANSFELD et al. in der Hochgebirgsklinik Davos-Wolfgang nachgewiesen [19]. Dosierungsempfehlungen: für Kinder von 0-1 Jahr 0,02-0,05 g, >1-4 Jahre 0,05-0,15 g, $>4-10$ Jahre 0,1-0,2 g und >10-16 Jahre 0,2-0,3 g. Gegenanzeigen, Neben- und Wechselwirkungen sind keine bekannt. Wegen der geringen Tagesdosierungen sind nur Extrakte empfohlen.

\section{Lichen islandicus (Isländisches Moos)} Indikationen sind Schleimhautreizungen im Mund- und Rachenraum und damit verbundener trockener Reizhusten [20]. Gegenanzeigen, Neben- und Wechselwirkungen sind nicht bekannt. Die Droge wird zerkleinert für Aufgüsse sowie für galenische Zubereitungen wie z.B. Pastillen ohne Zucker. Die Dosierung beträgt für Kinder $>1-$ 4 Jahre 1-2 g, >4-10 Jahre 2-4 g und >10-16 Jahre 4-6 g.

\section{Liquiritiae radix (Süssholzwurzel)}

Die kleingeschnittene, geschälte Droge aus der Süssholzwurzel, Drogenpulver, Trockenextrakte für Aufgüsse und Abkochungen werden bei Katarrhen der oberen Luftwege eingesetzt [21]. Gegenanzeigen sind cholestatische Lebererkrankungen, Hypertonie und Hypokaliämie. Die Dosierung beträgt für Liquiritiae radix >1-4 Jahre 1,5-3 g und >4-16 Jahre 3-5 g, für Liquiritiae radix-Succus >4-10 Jahre 0,1-0,5 g, >10-16 Jahre 0,5-1 g.

\section{Malvae flos/folium (Malvenblüten/-} blätter)

Die zerkleinerte Droge aus Malvenblüten/-blättern steht für Aufgüsse sowie andere galenische Zubereitungen bei Schleimhautreizungen im Mund- und Rachenraum und damit verbundenem trockenen Reizhusten zur Verfügung [22]. Neben- und Wechselwirkungen sowie Gegenanzeigen sind nicht bekannt. Die Dosierung für Malvae flos/ folium beträgt für Kinder von 0-1 Jahr 1 g, >1-4 Jahre 1-2 g, >4-10 Jahre 2$4 \mathrm{~g},>10-16$ Jahre 4-5 g.

\section{Matricariae flos (Kamillenblüten)}

Bei Haut- und Schleimhautentzündungen sowie entzündlichen Erkrankungen und Reizzuständen der Atemwege stehen flüssige und feste Darreichungsformen aus Kamillenblüten zur äusseren Anwendung bzw. zur Inhalation zur Verfügung [23]. Die Dosierung soll von 016 Jahre $3-10 \%$ betragen. Gegenanzeige ist eine Allergie auf Kamillenblüten.

\section{Menthae arvensis aetheroleum (Minzöl)}

Das ätherische Öl aus der Minze [24] kann bei Katarrhen der Atemwege inhalativ tropfenweise in heissem Wasser angewendet oder aufgetropft werden (Taschentuch, Bettzeug etc.). Bei Säuglingen und Kleinkindern dürfen Minzöl-Zubereitungen nicht im Gesicht, im Besonderen nicht an der Nase aufgetragen werden. Gegenanzeigen sind Gallensteinleiden, Verschluss der Gallenwege, Gallenblasenentzündung, schwere Leberschäden und sklerosierende Laryngitis. Die Dosierung beträgt bei Kindern >1-4 Jahre 1-3 Tr., >4-10 Jahre 2-4 Tr., >10-16 Jahre 3-6 Tr.

\section{Menthae piperitae aetheroleum (Pfefferminzöl)}

Das ätherische Öl aus der Pfefferminze wird wie bei der Minze topisch bei Katarrhen der Atemwege zur Inhalation in heissem Wasser oder auf das Bettzeug getropft angewandt [25]. Es gelten dieselben Gegenanzeigen wie bei der Minze. Die Dosierung beträgt bei Kindern von 0-1 Jahr 1 Tr., >1-4 Jahre 1- 2 Tr., >4-10 Jahre 2-3 Tr., >10-16 Jahre 3-4 Tr. Für Nasensalben gelten folgende Dosierungsempfehlungen: >1-4 Jahre 0,2-1\%, >410 Jahre 0,3- 2\%, >10-16 Jahre 1-3\%. 

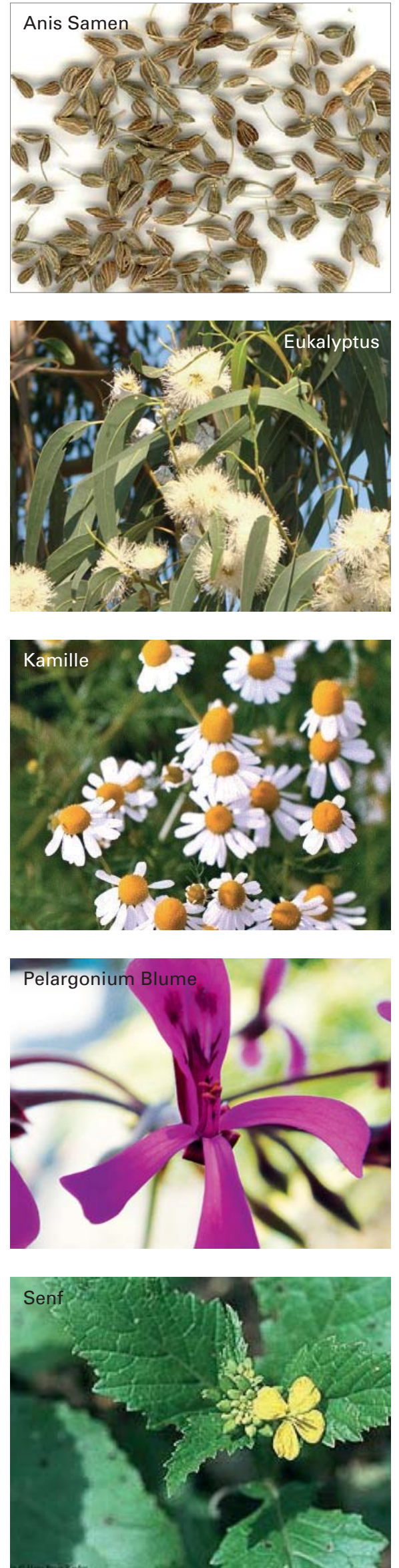
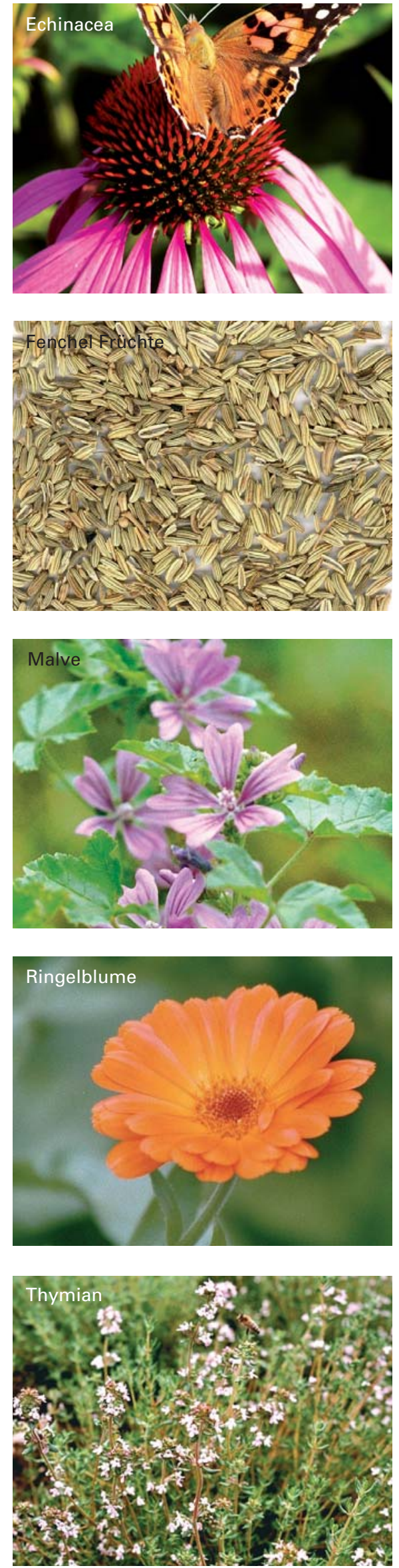
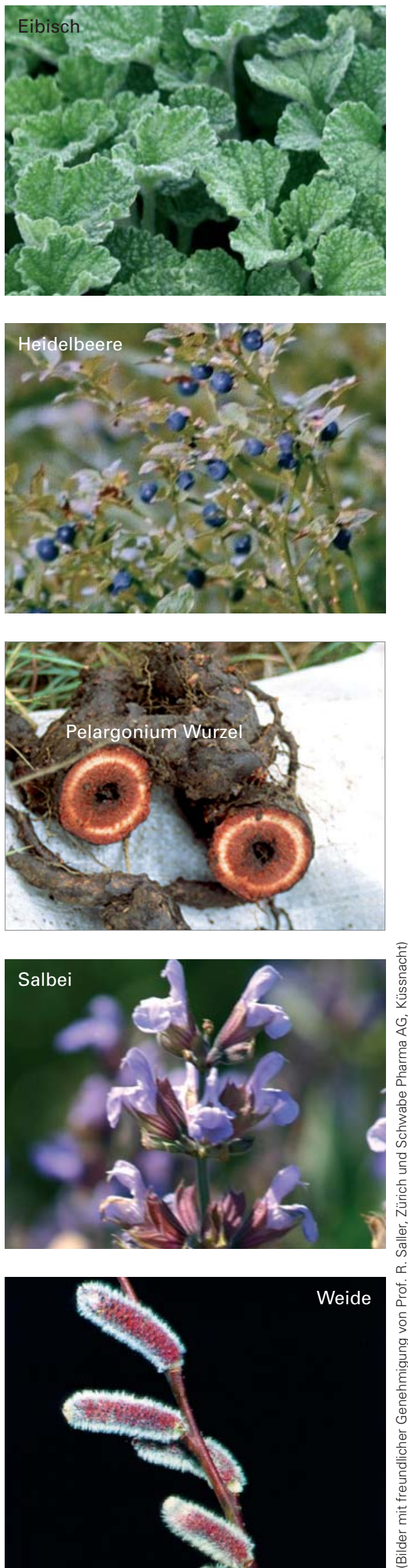

Abb. 1. Auswahl an Arzneipflanzen, die bei Infektionen der Atemwege eingesetzt werden. 


\section{Myrtilli fructus (Heidelbeeren)}

Bei leichten Entzündungen der Mundund Rachenschleimhaut steht die getrocknete Droge aus Heidelbeeren zum Kauen oder Abkochungen zum Einnehmen oder für Mundspülungen zur Verfügung [26]. Die Dosierung für die Abkochung über den Tag verteilt einzunehmen beträgt von 0-1 Jahr 5$10 \mathrm{~g},>1-4$ Jahre 10-15 g, >4-10 Jahre 15-20 g, >10-16 Jahre 20-60 g. Für die topische Applikation wird als Dosierung für die Abkochung empfohlen: 0-1 Jahr 5\%, >1-16 Jahre 10\%.

\section{Pelargonium sidoides}

Der Extrakt EPS ${ }^{\circledR} 7630$ aus Pelargonium-sidoides-Wurzel wirkt antimikrobiell, immunstimulierend und sekretolytisch $[27,28,29,30]$. Die Wirksamkeit bei Kindern ist in Studien belegt bei der akuten Bronchitis [31], der viralen Tonsillopharyngitis [32] und der Sinusitis [33]. Die Tagesdosierung beträgt bei Kindern von 1-5 Jahren 3x10 Tr., von 6-12 Jahren 3x20 Tr. und >12 Jahren $3 \times 30$ Tr..

\section{Piceae aetheroleum (Fichtennadelöl)}

Fichtennadelöl wird in der Pädiatrie in Tropfenform auf 11 heisses Wasser zur Inhalation bei katarrhalischen Erkrankungen der oberen und unteren Atemwege gegeben [34]. Die Dosierung beträgt für Kinder von 0-1 Jahr 2 Tr. auf 11 heisses Wasser, >1-4 Jahre 2-4 Tr., >4-16 Jahre 3-4 Tr. Als Nebenwirkungen können an Haut und Schleimhäuten verstärkte Reizerscheinungen auftreten. Bronchospasmen können verstärkt werden. Selten tritt eine Kontaktdermatitis auf. Gegenanzeigen sind Keuchhusten, bronchiale Hyperreagibilität und Asthma bronchiale. Bei peroraler Applikation von frischen Fichtennadelspitzen (Piceae turiones recentes) bestehen diese Gegenanzeigen hingegen nicht. Die Dosierung beträgt bei Kindern >1-4 Jahre 1-2 g, >4-10 Jahre 2-4 g, >10-16 Jahre 5-6 g.

\section{Pini aetheroleum/turiones (Kiefernnadelöl, -sprossen)}

Auch Kiefernadelöl darf nicht bei Asthma und Keuchhusten angewandt werden; Bronchospasmen können verstärkt werden und es können Reizerscheinungen an Haut und Schleim- häuten auftreten. In der Pädiatrie wird es bei katarrhalischen Erkrankungen der oberen und unteren Luftwege inhalativ [35] in folgenden Dosierungen angewandt: Bei Kindern von 0-1 Jahr 1-2 Tr. auf das Kopfkissen, >1-4 Jahre 2-3 Tr. auf das Kopfkissen, >4-16 Jahre 3-4 Tr./l heisses Wasser inhalativ. Bei Kiefernsprossen sind keine Gegenanzeigen oder Nebenwirkungen bekannt. Sie werden peroral appliziert [36]. Die Dosierung beträgt bei Kindern von 0-1 Jahr 1-2 g, >4-10 Jahre 1-3 g, >10-16 Jahre 2-3 g.

\section{Plantaginis lanceolatae herba (Spitzwegerichkraut)}

Die zerkleinerte Droge von Spitzwegerichkraut sowie andere galenische $\mathrm{Zu}$ bereitungen werden peroral bei Katarrhen der Luftwege und entzündlichen Veränderungen der Mund- und Rachenschleimhaut gegeben [37]. Gegenanzeigen, Neben- und Wechselwirkungen sind nicht bekannt. Die Dosierung beträgt bei Kindern $>1-4$ Jahre 1-2 g, >4-10 Jahre 2-4 g, >10-16 Jahre $3-6 \mathrm{~g}$.

\section{Primulae flos/radix}

\section{(Schlüsselblumenblüten, -wurzel)}

Die zerkleinerte Droge von Schlüsselblumenblüten wird für Aufgüsse und andere galenische Zubereitungen peroral bei Katarrhen der Atemwege gegeben [38]. Gegenanzeige ist eine bekannte Allergie gegen Primel. Vereinzelt können Magenbeschwerden und Übelkeit auftreten. Die Dosierung beträgt bei Kindern von 0-1 Jahr 0,5-1 g, >1-4 Jahre 1-2 g, >4-10 Jahre 2-3 g, $>10-16$ Jahre 2-4 g. PrimelwurzelDroge wird wie folgt dosiert: 0-1 Jahr 0,05-0,3 g, >1-4 Jahre 0,2-0,6 g, >416 Jahre $0,5-1 \mathrm{~g}$.

\section{Salicis cortex (Weidenrinde)}

Bei fieberhaften Erkrankungen und Kopfschmerzen werden Drogen aus Weidenrinde per os gegeben [39]. Neben- und Wechselwirkungen sowie Gegenanzeigen entsprechen denen bei Salicylaten aufgrund des Salicins in der Weidenrinde. Die Dosierung beträgt bei Kindern >1-4 Jahre $3 x$ tgl. $5-10 \mathrm{mg}$ Gesamtsalicin, >4-10 Jahre 3x tgl. 1020 mg, >10-16 Jahre $3 x$ tgl. 20-40 mg.

\section{Sambuci flos (Holunderblüten)}

Holunderblüten werden bei Erkältungskrankheiten unzerkleinert für Teeaufgüsse (mehrmals täglich 1-2 Tassen trinken) angewendet [40]. Gegenanzeigen, Neben- und Wechselwirkungen sind nicht bekannt. Die Dosierung beträgt für Kinder von 0-1 Jahr 1-2 g, $>1-4$ Jahre 2-5 g, >4-10 Jahre 5-10 g, >10-16 Jahre 10-15 g.

\section{Thymi herba (Thymiankraut)}

Indikationen sind Bronchitis, Keuchhusten, Katarrhe der oberen Atemwege [41]. Neben- und Wechselwirkungen sowie Gegenanzeigen sind nicht bekannt. Die Dosierung für Aufgüsse: für Kinder von 0-1 Jahr 0,5-1 g/Tasse, $>1-16$ Jahre 1-2 g/Tasse. Für den Fluidextrakt per os ist die Dosierung für Kinder >1-4 Jahre 0,3-1 g, >4-10 Jahre 0,5-1,3 g, >10-16 Jahre 1-2 g.

\section{Tiliae flos (Lindenblüten)}

Die zerkleinerte Droge aus Lindenblüten wird bei Erkältungskrankheiten und damit verbundenem Husten für Teeaufgüsse sowie andere galenische Zubereitungen per os angewendet [42]. Gegenanzeigen, Neben- oder Wechselwirkungen sind nicht bekannt. Die Dosierung beträgt bei Kindern $>9$ Monate -1 Jahr 0,2-1 g, >1-4 Jahre 1-2 g, >416 Jahre 2-4 g.

\section{Verbasci flos (Wollblumen)}

Indikation für die zerkleinerte Droge aus Wollblumen für Aufgüsse sowie für andere galenische Zubereitungen zum Einnehmen sind Katarrhe der Atemwege [43]. Neben- und Wechselwirkungen sowie Gegenanzeigen sind nicht bekannt. Die Dosierung beträgt für Kinder von 0-1 Jahr 0,5-1 g, >1-4 Jahre 1-2 g, $>4-10$ Jahre 2-3 g, >10-16 Jahre 3-4 g.

\footnotetext{
Literatur

1 Committee for Proprietary Medicinal Products (CPMP): Note for Guidance on Clinical Investigation of Medicinal Products in Children. Revision 1, Draft 5, 16. 7. 1996.

2 Schwabe D, Staib AH: Arzneimittel im Kindesalter. In: Rietbrock N, Staib AH, Loew D (Hrsg.): Klinische Pharmakologie. 3. Aufl. Darmstadt: Steinkopff; 1996.

3 Schilcher $\mathrm{H}$ : Phytotherapie in der Kinderheilkunde - Handbuch für Ärzte und Apotheker. 2. Aufl. Stuttgart: Wissenschaftliche Verlagsgesellschaft; 1992.

4 Kooperation Phytopharmaka (Hrsg.): Kinderdosierungen von Phytopharmaka. 3. Aufl. Bonn: Kooperation Phytopharmaka; 2002.
} 
5 Wichtl M (Hrsg.): Teedrogen und Phytopharmaka. 3. Aufl. Stuttgart: Wissenschaftliche Verlagsgesellschaft; 1997

6 Monographien: Althaeae folium (Eibischblätter), Altheae radix (Eibischwurzel). BAnz. Nr. 43 vom 2.3.1989.

7 Monographie: Anisi fructus (Anisfrüchte) BAnz. Nr. 122 vom 6.7.1988

8 Monographie: Anisi stellati fructus (Sternanisfrüchte). BAnz. Nr. 122 vom 6.7.1988.

9 Monographie: Arnicae flos (Arnikablüten). BAnz. Nr. 228 vom 5.12.1984.

10 Monographie: Calendulae flos (Ringelblumenblüten). BAnz. Nr. 50 vom 13.3.1986.

11 Monographie: Caryophylli flos (Gewürznelken). BAnz. Nr. 223 vom 30.11.1985.

12 Monographie: Echinaceae purpureae herba. BAnz. Nr. 43 vom 2.3.1989.

13 Monographie: Eucalypti folium (Eucalyptusblätter). BAnz. Nr. 177 a vom 24. 9. 1986, BAnz. Nr. 50 vom 13.3.1990.

14 Monographie: Farfarae folium (Huflattichblätter). BAnz. Nr. 138 vom 27.7.1990

15 Monographie: Filipendula ulmaria (Mädesüß). BAnz. Nr. 43 vom 2.3. 989.

16 Monographie: Foeniculi fructus (Fenchelfrüchte). BAnz. Nr. 74 vom 19.4.1991.

17 Monographie: Hederae helicis folium (Efeublätter). BAnz. Nr. 122 vom 6.7.1988.

18 Meyer-Wegener J, Liebscher K, Hettich M: Efeu versus Ambroxol bei chronischer Bronchitis. Z Allgemeinmed 1993;69:61-66.

19 Mansfeld HJ, Höhre H, Repges R, Dethlefsen $\mathrm{U}$ : Therapie des Asthma bronchiale mit Efeublätter-Trockenextrakt. Münch Med Wochenschr 1998;140(3):26-30.

20 Monographie: Lichen islandicus (Isländisches Moos). BAnz. Nr. 43 vom 23.1989.

21 Monographie: Liquiritiae radix (Süßholzwur- zel). BAnz. Nr. 90 vom 15.5.1985.

22 Monographie: Malvae flos, Malvae folium. BAnz. Nr. 43 vom 2.3.1989.

23 Monographie: Matriaciae flos (Kamillenblüten). BAnz. Nr. 228 vom 5.2.1984, BAnz. Nr. 50 vom 13.3.1990.

24 Monographie: Menthae arvensis aetheroleum (Minzöl). BAnz. Nr. 50 vom 13.3.1990.

25 Monographie: Menthae piperitae folium (Pfefferminzblätter). BAnz. Nr. 223 vom 30.11. 1985.

26 Monographie: Myrtilli fructus (Heidelbeeren). BAnz. Nr. 76 vom 23.4.1987.

27 Kayser O, Kolodziej H: Antimicrobial activity of extracts and constituents of Pelargonium sidoides and Pelargonium reniforme. Planta Med 1997:63:508-510.

28 Kayser O, Kolodziej $H$, Kiderlen AF: Immunomodulatory principles ofPelargonium sidoides. Phytother Res 2001:15:122-126.

29 Kolodziej $\mathrm{H}$, Kayser O, Radtke OA Kiderlen AF, Koch E: Pharmacological profile of extracts of Pelargonium sidoides and their constituents. Phytomedicine 2003; 10 (Suppl. 4): $18-24$

30 Conrad A, Hansmann C, Engels I, Daschner FD, Frank U: Extract of Pelargonium sidoides (EPS ${ }^{\circledR}$ 7630) improves phagocytosis, oxidative burst, and intracellular killing of human peripheral blood phagocytes in vitro. Phytomedicine 2007;14 (Suppl. 6):46-51.

31 Matthys H, Kamin W, Funk P, Heger M: Pelargonium sidoides preparation (EPS ${ }^{\circledR} 7630$ ) in the treatment of acute bronchitis in adults and children. Phytomedicine 2007;14 (Suppl. 6):69-73

32 Bereznoy VV, Riley DS, Wassmer G, Heger $\mathrm{M}$ : Efficacy of Pelargonium sidoides in children with acute non-group A beta-hemolytic streptococcus tonsillopharyngitis: a randomized, double-blind, placebo-controlled trial. Altern Ther Health Med 2003;9:68-79.

33 Schapowal A, Heger M: EPs® 7630 Lösung (Umckaloabo ${ }^{\circledR}$ ) bei Sinusitis. Z Phytotherapie 2007:28:58-65.

34 Monographie: Piceae aetherolum (Fichtennadelöl). BAnz. Nr. 154 vom 21.8.1985.

35 Monographie: Pini aetheroleum (Kiefernnadelöl). BAnz. Nr. 154 vom 31.8.1985.

36 Monographie: Pini turiones (Kiefernsprossen). BAnz. Nr. 173 vom 18.9.1986 und BAnz. Nr. 50 vom 13.3.1990.

37 Mongraphie: Plantaginis lanceolatae herba (Spitzwegerichkraut). BAnz. Nr. 223 vom 30 11.1985

38 Monographie: Primulae flos (Schlüsselblumenblüten). BAnz. Nr. 50 vom 13.3.1990.

39 Mongraphie: Salicis cortex (Weidenrinde). BAnz. Nr. 228 vom 5.12.1984.

40 Monographie: Sambuci flos (Holunderblüten). BAnz. Nr. 50 vom 13.3.1986.

41 Monographie: Thymi herba (Thymiankraut).

42 Monographie: Tiliae flos (Lindenblüten). BAnz. Nr. 164 vom 1.9.1990.

43 Monographie: Verbasci flos (Wollblumen). BAnz. Nr. 22 vom 1.2.1990.

\section{Anschrift des Autors}

PD Dr. med. Dr. h.c. Andreas Schapowal Facharzt für Hals-Nasen-Ohren-Heilkunde Facharzt FMH für Allergologie und klinische Immunologie

Hochwangstrasse 3, CH-7302 Landquart andreas@schapowal.ch

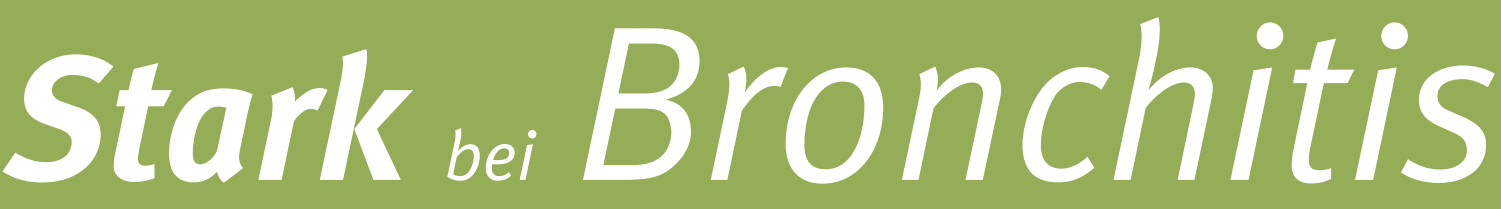
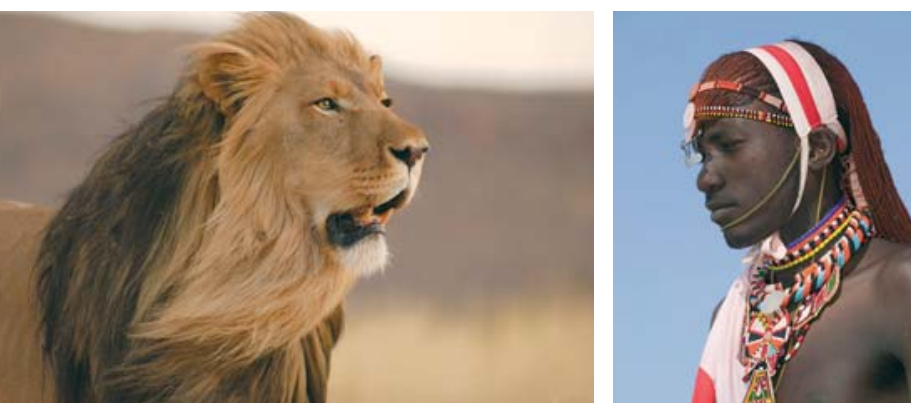

Umckaloabo Infektabwehr aus der Natur

Die gezielte Lösung bei viralen Atemwegserkrankungen mit 3-fach Wirkung

- gegen Viren

- gegen Bakterien ${ }^{2,3}$

- sekretomotorisch ${ }^{4}$

Umckaloabo ${ }^{\circledR}$ - der Spezialextrakt zur effizienten Behandlung einer akuten Bronchitis. Wissenschaftliche Studien belegen die klinische Wirksamkeit von Umckaloabo ${ }^{\circledR}$ für Erwachsene und Kinder ab 1 Jahr.

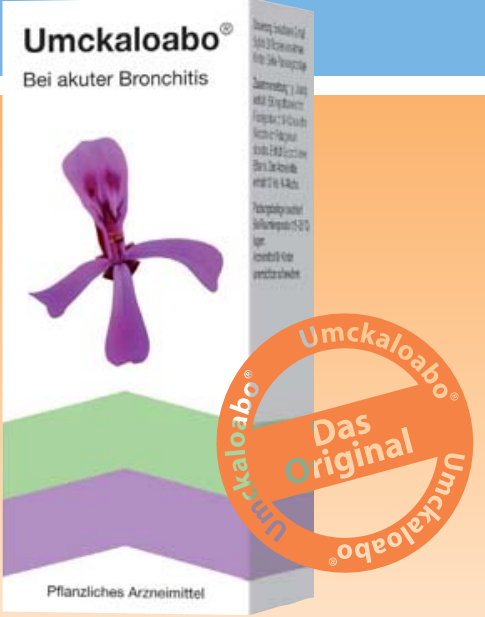

Referenzen:

1 Kolodziej et al. Umckaloabo ${ }^{\oplus}$ - from traditional application to modern phytodrug. DAZ 2003 (143/12) 55-64 2 Kolodziej et al. In vitro evaluation of antibacterial and immunmodulatory activities of Pelargonium sidoides and the related herbal drug preparation EPs ${ }^{\circledast} 76303$ Kayser et al. Antibacterial Activity of Extracts and Constituents of Pelargonium sidoides and Pelargonium reniforma. Pharm Pharmacol Lett 7 (1997) 2/3; $71-744$ Neugebauer et al. A new approach to pharmacological effects on ciliary beat frequency in cell cultures - exemplary measurements under Pelargonium sidoides extract (EPs $\left.{ }^{\circledast} 7630\right)$. Phytomedecine 12 (2005) 46-51 5 Arzneimittelkompendium der Schweiz 